This is an Accepted Manuscript of an article published by Taylor \& Francis Group in Infancia y Aprendizaje, available online: https://doi.org/10.1174/021037013808200302 


\title{
Contenidos y modos conversacionales en adolescentes. Debatiendo sobre conflictos grupales y violencia de pareja
}

\author{
Miguel J. Bascón, Samuel Arias, Manuel L. De la Mata \\ Universidad de Sevilla
}

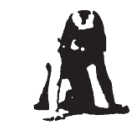

Resumen

La adolescencia es un periodo de expansión de las relaciones y redes sociales, e inherentemente, de aumento de las situaciones conflictivas y violentas. Por ello, nuestro objetivo es analizar la gestión discursiva de conflictos grupales y violencia de pareja en adolescentes, explorando los modos conversacionales y los contenidos que articulan la conversación durante el afrontamiento de las situaciones controvertidas. Mediante una aproximación analítica que combina técnicas estadísticas y cualitativas, estudiamos el discurso de 42 chicos y chicas entre 14 y 17 años, debatiendo en grupos de discusión sobre dilemas. Los resultados muestran el efecto combinado de las variables tipo de grupo y tipo de dilema sobre los modos conversacionales de gestión, destacando el disputativo (imposiciones, mandatos...) cuando chicos y chicas debaten juntos sobre un episodio de violencia de género. Referente a los contenidos, se observan mayores diferencias temáticas entre chicos y chicas ante la violencia de pareja, evidenciándose el carácter situado del discurso; así, los chicos no visibilizan el maltrato mientras que las chicas asumen responsabilidad. Finalmente, se ofrecen algunas consideraciones para la práctica aplicada.

Palabras clave: Adolescencia, discurso, dilema, resolución de conflicto, violencia.

\section{Contents and conversational modes in adolescents: Debating group conflicts and partner violence}

\begin{abstract}
Adolescence as a period is associated to the extension of social relationships and networks, and, inherently, to an increase of conflicts and situations of violence. Therefore, our aim is to analyse the discursive management of group conflicts and partner violence in adolescents, exploring conversational modes and contents that articulate conversation during the discussion of controversial situations. Using an analytical approach that combines statistical and qualitative techniques, we studied the discourse produced by 42 boys and girls (aged 14 to 17 years), during group discussion of dilemmas. The results evidence the combined effect of the variables type of group and type of dilemma on the conversational modes of conflict management, with a predominance of the disputative form (impositions, commands...) when boys and girls discuss together about an episode of gender violence. Regarding the contents, there are larger thematic differences between boys and girls to partner violence, providing some evidence of the situated character of the discourse; so, boys do not recognize maltreatment while girls take responsibility. Finally, some considerations for applied practice are presented.

Keywords: Adolescence, discourse, dilemma, conflict resolution, violence.
\end{abstract}

Correspondencia con los autores: Departamento de Psicología Experimental. Universidad de Sevilla. C/ Camilo José Cela s/n. 41018 Sevilla. E-mails: mjbascon@us.es-Samuel@us.es -mluis@us.es Original recibido: 19 de junio de 2012. Aceptado: 12 de abril de 2013. 
Infancia y Aprendizaje, 2013, 36 (4), pp. 489-506

El objetivo que guía este trabajo es analizar la gestión discursiva de situaciones interpersonales controvertidas en adolescentes, concretamente episodios de conflicto en grupos de iguales y de violencia de pareja, explorando los modos conversacionales y los contenidos que subyacen y orientan la conversación durante el afrontamiento de los mismos.

Comenzamos considerando la adolescencia como una etapa del ciclo vital caracterizada y condicionada por las numerosas transformaciones que se producen a nivel físico, psicológico y social (Garaigordobil, 2012). Este momento evolutivo tiene una importante repercusión sobre el desarrollo, la salud y el ajuste psicosocial de las personas, ya que es determinante en la consolidación de las habilidades sociales, los procesos psicológicos, la expansión de relaciones entre iguales, el fraguado de nuevas redes sociales y la constitución de grupos y parejas sentimentales. El tránsito por la adolescencia puede verse condicionado por factores de riesgo de distinta índole y procedencia: culturales, ambientales, relacionales, personales, etcétera, que a veces desembocan en conflictos y violencia. Como señalan Inglés et al. (2008), la adolescencia es un período en el que aumentan de forma significativa diferentes tipos de manifestaciones conflictivas y violentas. En este trabajo nos centramos principalmente en situaciones interpersonales como los conflictos de grupo y la violencia de pareja.

Un aspecto a tener en cuenta a la hora de hablar de estos comportamientos es la diferenciación entre conflicto y violencia. Jares (2001) considera el conflicto como un proceso de incompatibilidad y desacuerdo entre partes que perciben intereses opuestos. Respecto a la violencia, Prieto, Carrillo y Jiménez (2005) la definen como una violación o arrebato al ser humano de algo que le es esencial, ya sea físico, psíquico, moral, etcétera.

Ortega y Mora (2008) indican que el conflicto es un fenómeno natural de la confluencia social y confrontación de motivos e intereses, mientras que la violencia no es puntual, sino que implica un proceso de desequilibrio en el que la víctima es dominada por el agresor. Por tanto, conflicto y violencia no deben ser empleados como procesos análogos. En este sentido, diferenciaremos dos de los ámbitos de relación más típicos durante la adolescencia como son los grupos o pandillas y las relaciones de pareja. En las pandillas los conflictos que giran sobre la aceptación y rechazo entre iguales son uno de los focos problemáticos más frecuentes, mientras que en las relaciones de noviazgo muchos casos de violencia parecen originarse en torno a la sexualidad y al uso del poder.

La aproximación al estudio de la gestión y afrontamiento de los conflictos y la violencia en adolescentes es un aspecto nuclear en nuestro trabajo. Para ello nos apoyamos en el estudio de los procesos psicológicos a través del discurso, entendido como una acción mediada por el lenguaje (Wertsch, 1991). Partimos del correlato existente entre discurso y pensamiento, de manera que la estructura del discurso puede revelarnos la estructura del pensamiento (Billig, 1987). El análisis de los discursos proferidos permite conocer la función de las intervenciones y turnos de palabra durante las interacciones, es decir, el papel que cada enunciado tiene en la conversación (Quignard, 2005).

En este sentido Bakhtin (1986) considera el enunciado como la unidad real de la comunicación con límites determinados por el cambio de hablante o turno de palabra. Así, todo enunciado hace referencia a un determinado contenido semántico y siempre va dirigido a alguien explícita o implícitamente (addressivity). Esta dimensión del discurso es denominada organización retórica o argumentativa (Edwards, 1996; Potter y Wetherell, 1995); supone que una producción lingüística nunca es neutra, siempre se realiza desde una determinada perspectiva que trata de ser presentada como alternativa válida dentro de la conversación. El enunciado es por tanto considerado un eslabón en la cadena de la comunicación, pudiéndose dar como respuesta a enunciados previos o como anticipo de otros.

En nuestro trabajo, planteamos situaciones de debate para que chicos y chicas conversaran y discutieran sobre conflictos de pandilla y violencia en la pareja. Eyre, Davis y Peacock (2001) examinaron el contenido, la estructura retórica y la función 
de la argumentación en 150 adolescentes conversando sobre sexo y, comparando enfoques de análisis, concluyeron que el estudio del discurso resultó el procedimiento más exitoso para la identificación de ideas subyacentes relacionadas con conflictos de género. Kuhn, Wang y Li (2010) en base a una investigación con alumnado de diferentes niveles educativos y culturas como la estadounidense y la china, resaltaron las ventajas de la discusión y el debate en grupo en relación con un tema que involucre puntos de vista opuestos.

Durante la conversación el debate cobra un papel destacado, ya que los significados son continuamente negociados y renegociados (Mercer, 2001). Su carácter social y retórico propicia la controversia y permite que las acciones se manifiesten. La propuesta de Mercer (2001) en relación con los distintos modos de conversación resulta un instrumento válido para el estudio de los procesos discursivos durante el afrontamiento de episodios controvertidos. En su afán por el estudio de los mecanismos de construcción compartida de significados durante la discusión propone la existencia de tres modos de conversación, con propiedades diferenciales. En la conversación disputativa se produce un intercambio de turnos caracterizados por la negación y oposición para aceptar el punto de vista del otro, y por la constante reafirmación de la posición propia que dificulta el acuerdo. Lo contrario sucede en la conversación acumulativa, en la que se mediante una aceptación no crítica de la otra parte se llega a una perspectiva compartida. Finalmente, en la conversación exploratoria se aborda de una manera evaluativa, razonada, consensuada y constructiva las ideas de los demás, rebatiendo y apoyando las propuestas.

Resulta loable estudiar cómo se utilizan estos tres tipos de conversación durante la discusión de situaciones controvertidas. Así, por un lado parece interesante la influencia de aspectos como el tipo de problema abordado y el sexo, y por otro, los contenidos temáticos tratados. Con respecto a lo primero, Crandall, Tsang, Goldman y Pennington (1999) plantean la relación entre contenido de un dilema y usos discursivos, y Leman y Duveen (2003) entre estilo conversacional y sexo de la persona con la que se discute. Referente a las temáticas, Sastre, Arantes y González (2007) muestran cómo en la adolescencia no se identifica ni se interpreta claramente la violencia de género, produciéndose una tendencia a disculpar y minimizar los actos violentos. Sobre este fenómeno, Díaz-Aguado (2003) observó que eran muchas más las chicas adolescentes que rechazaban el uso de la violencia y muchos más los chicos que lo justificaban culpando frecuentemente a la víctima.

Según Gilligan (1982) estas diferencias en las formas de interpretar la violencia pudieran estar relacionadas con diferentes éticas, al observar la existencia de una orientación hacia la responsabilidad o ética de cuidado, preferentemente en las mujeres, que surge de la conciencia de formar parte de una red de relaciones y que adopta el punto de vista del "otro concreto", sus necesidades particulares, sentimientos y motivaciones. Esta manera de entender el mundo difiere de la ética de justicia que canaliza, mediante derechos y normas imparciales, sobre todo las acciones de los hombres. No obstante, la propia autora en una obra posterior (Gilligan, 1993) aclara que esta distinción no se sustenta en fundamentos esencialistas, sino que ambas éticas se complementan, relacionan e integran de cara al desarrollo.

Queremos resaltar que la utilización de la variable sexo podría inducir a una visión esencialista, homogénea y dicotómica. Por el contrario, adoptamos el enfoque Doing Gender (West y Zimmerman, 1987) que enfatiza la construcción social del género desde niveles socioculturales, relacionales e individuales, desencadenándose una "profecía autocumplida" (Crawford y Chaffin, 1997) o comportamiento en consonancia con lo esperado.

En definitiva, el propósito general de este trabajo se orienta hacia explorar la gestión discursiva de episodios de conflicto de pandilla y de violencia de pareja en adolescentes. Trataremos de aproximarnos mediante el estudio de los usos conversacionales de chicos y chicas adolescentes, durante la discusión y debate sobre dilemas. Se establecen dos objeti- 
vos: analizar el posible efecto del tipo de grupo y del tipo de dilema sobre los modos conversacionales (acumulativo, disputativo y exploratorio), e identificar los principales contenidos temáticos manifestados por chicos y chicas.

Para dar respuesta al primer objetivo planteamos la siguiente hipótesis: esperamos encontrar efectos significativos del tipo de grupo y del tipo de dilema sobre los modos conversacionales. Concretamente, un mayor número de intervenciones de tipo disputativo en los grupos de chicos y chicas debatiendo juntos sobre violencia de pareja, debido a diferencias en la interpretación y visibilización del maltrato de género; ello dificultará la resolución del episodio, es decir, la consecución negociada de una postura relativamente compartida. Respecto al segundo objetivo, queremos comprobar si las diferencias en las temáticas tratadas por chicos y chicas pueden asociarse con diferentes maneras de interpretar situaciones de violencia y/o con diferencias en las éticas subyacentes.

\section{Método}

\section{Participantes}

Participaron en el estudio 42 adolescentes de nacionalidad española correspondientes a dos aulas completas, 23 chicas y 19 chicos. La diferencia en el número se debió a la mayor presencia de chicas en las aulas y a las ausencias del día de la recogida de datos; además se evitó igualar los números mediante descartes por sus posibles efectos negativos. Seleccionamos edades entre 14 y 17 años para tener una representación más amplia del rango de edad en el que parece incrementarse el número de situaciones conflictivas y violentas. Cursaban tercer y cuarto curso de Educación Secundaria Obligatoria en un Instituto de Enseñanza Pública de la ciudad de Sevilla, ubicado en zona urbana de nivel socioeconómico medio.

Para la formación de los grupos de discusión los y las participantes fueron seleccionados sobre la base de un criterio de heterogeneidad en función de las posiciones mostradas ante una tarea de exploración de ideas previas sobre conflicto grupal y violencia de pareja (Fase $1 \mathrm{del}$ procedimiento). Se realizaron debates con la totalidad del grupo-clase en los que se presentaron noticias y situaciones relacionadas con estos dos temas para que chicos y chicas opinaran sobre ellos. Las posiciones fueron medidas en función del grado de identificación del conflicto y la violencia, que osciló entre la visibilización y la no visibilización, pasando por estados intermedios de identificación parcial. Esta horquilla permitió que todo el alumnado quedase ubicado en uno u otro nivel, y sirvió de base para garantizar que los diferentes niveles de representación generaran mayor confrontación durante el debate.

\section{Materiales}

Se elaboraron dos dilemas o situaciones hipotéticas pero verosímiles y cercanas a la cotidianidad adolescente. Uno hacía referencia a un conflicto de intereses en pandilla y el otro a violencia de género. Se procuró mayor gravedad en el desenlace del episodio de maltrato en pareja y que la persona agresora fuese solo un chico buscando aumentar su visibilización y el impacto emocional. La construcción de los dilemas se llevó a cabo teniendo en cuenta los contenidos más comentados en la Fase 1 del procedimiento y en base a la literatura científica.

Se manejaron dos versiones homólogas del dilema de pandilla, una con un chico como protagonista y otra con una chica. Cada versión se planteó a cuatro grupos diferentes, uno de chicos y otro de chicas debatiendo por separado y dos haciéndolo juntos. El dilema de violencia de pareja tenía únicamente a un chico como maltratador, y se administró a los ocho grupos, cuatro de un tipo (dos de chicas y dos de chicos por separado) y cuatro del otro (debatiendo juntos). En todos los casos se les pidió que intentaran resolver la controversia, para observar los modos de conversación utilizados durante la gestión de los problemas. Por tanto, cada grupo realizó dos debates, uno en base a cada dilema: 
Discusión sobre el dilema 1. Conflicto de pandilla

\begin{abstract}
Se trata de una pandilla de amigos/as que se conocen desde hace muchos años. Todo va bien por la gran amistad que les une. Un día alguien comenta que su primo/a va a pasar el verano en casa, y que como el año anterior vendrá al grupo. Rápidamente, un/a chico/a se niega porque esta persona intentó quietarle su pareja, en cambio el resto aceptan que venga. La situación genera una discusión en el grupo.
\end{abstract}

Discusión sobre el dilema 2. Violencia de pareja

En una pareja, el chico continuamente está ridiculizando, burlando y menospreciando a su novia, a solas y delante de los demás. Las críticas son referentes a su aspecto físico, su forma de vestir, etc. La chica va disminuyendo su autoestima y tras varios meses sufriendo entra en una depresión que le conduce al suicidio. La familia denuncia al novio por incitación y maltrato, y lo acusa de ser el responsable de la muerte.

\title{
Procedimiento
}

Tras el preceptivo protocolo de acceso y consentimiento informado, el estudio se dividió en dos fases:

Fase 1. Exploración de ideas previas. Chicos y chicas realizaron una tarea de posicionamiento y opinión sobre acontecimientos y noticias relacionadas con los temas que se iban a tratar posteriormente en los debates grupales. Esta tarea permitió conocer los puntos de vista, ideas previas y diferente grado de identificación y visibilización de los conflictos de pandilla y la violencia de género en adolescentes, y así llevar a cabo la formación de los grupos de discusión de la forma más heterogénea posible para generar controversia en el debate.

Fase 2. Grupos de discusión y debate. Los y las adolescentes fueron organizados en ocho grupos de discusión, formados por un número de entre cuatro y seis miembros cada uno. En cuatro grupos, chicas (dos) y chicos (dos) debatieron por separado, y en otros cuatro lo hicieron juntos. A cada grupo se le planteó la tarea de debatir y discutir sobre los dos dilemas desarrollados para esta investigación. Fueron presentados por un investigador cuando se trataba de grupos de chicos, por una investigadora cuando se trataba de chicas, y cuando debatían juntos estaban presentes ambos. La presentación de los dilemas se realizó de manera oral (leídos) y los investigadores sólo intervinieron cuando fue preciso reconducir la conversación, por alejamiento del tema central planteado.

Se realizó un registro audio-visual de los debates, que posibilitó que el discurso proferido fuese digitalizado mediante el programa informático Adobe Premiere. Para la transcripción se empleó el programa Transana. El análisis estadístico de los modos conversacionales se realizó con el paquete SPSS y el abordaje cualitativo mediante el software Nud*IstNVivo.

\section{Análisis}

El conjunto de datos registrado fue sometido a un doble análisis. Se aplicó un tratamiento cuantitativo para delimitar los modos conversacionales en función de dos variables (primer objetivo), y posteriormente se llevó a cabo un análisis cualitativo para establecer los principales contenidos temáticos debatidos (segundo objetivo).

Modos conversacionales. Diseño y variables del análisis estadístico. Se consideraron tres variables; el tipo de grupo con dos condiciones, chicas-chicos debatiendo por separado y chicaschicos debatiendo juntos, el tipo de dilema con otras dos condiciones, conflicto de pandilla y violencia de pareja, y la modalidad conversacional con tres categorías de análisis derivadas de la propuesta de Mercer (2001):

Conversación disputativa: negación de lo ajeno y afirmación propia. Ejemplo:

1378: am: si has matado por mucho que haga tu abogado vas a la cárcel

1379: ac: hombre no es matarla

1380: am: has provocado...

1381: ac: ique no estoy matando! 
494 Infancia y Aprendizaje, 2013, 36 (4), pp. 489-506

Conversación acumulativa: aceptación poco crítica de lo ajeno. Ejemplo:

846. ra: yo lo hubiese dejado

847. pa: y yo

848. es: pero ya hace tiempo

849. ra: pero es que vamos hace tiempo

Conversación exploratoria: tratamiento crítico y constructivo las ideas. Ejemplo:

879. es: ¡me meto y más si es una amiga muy íntima y no se da cuenta, yo ahí me meto!

880. mb: tú piensa, tu novio y tú y ahora que una amiga se meta, que te aconseje pero que no se meta porque es peor

881. pa: además acabas tú peor, quedas mal con tu amiga y con el novio

El cálculo de la fiabilidad (Kappa de Cohen) indicó un muy buen nivel de acuerdo entre observadores $(k=0,88)$. Previamente, se procedió a un entrenamiento de los mismos para garantizar que el registro y la codificación de los datos se efectuaban bajo criterios consensuados.

Contenidos conversacionales. Codificación de temáticas en el análisis cualitativo. El tratamiento cualitativo de los datos se realizó mediante un análisis de aquellos enunciados cuyos contenidos se referían hacia un mismo tema, revelando la presencia de una serie de temáticas que emergen durante la discusión y que articulan el debate. Los mismos no estaban predeterminados a priori para su posterior observación y registro, sino que fueron detectados, delimitados y constituidos "en proceso" o "in vivo" a partir del discurso proferido por los y las participantes en los grupos de discusión. Las temáticas son cruzadas con los tres modos conversacionales planteados en el análisis anterior.

En este caso, la fiabilidad vino dada por la semejanza y concordancia en los contenidos temáticos detectados por los diferentes investigadores que analizaron los discursos.

\section{Resultados}

\section{Análisis estadístico de los modos conversacionales}

Tras registrar qué tipos de conversación emplean los y las adolescentes categorizando cada enunciado como acumulativo, disputativo o exploratorio, establecemos la significación estadística de los efectos entre las variables, es decir, la relación entre el tipo de grupo y tipo de dilema y la modalidad conversacional. El enunciado fue la unidad de análisis, considerado como el turno completo de palabra y unidad real de la comunicación (Bakhtin, 1986). Fueron analizados un total de 2346 enunciados que constituyen la matriz de datos. El proceso de análisis y exposición de los resultados cuantitativos es el siguiente. En primer lugar se exponen los datos relativos a la distribución agrupada de las tres variables, y en segundo lugar mostramos los valores estadísticos $(F)$ de los efectos así como su probabilidad $(p)$. Para el tratamiento de los datos se ha empleado la prueba Análisis de la Varianza Univariante (UNIANOVA).

Los datos combinados entre las tres variables (Tabla I) indican que la mayor producción discursiva tiene lugar cuando los y las adolescentes afrontan el dilema de violencia de género en los grupos en los que debaten juntos (frecuencia total $=847$ ). En estos escenarios, es principalmente la conversación disputativa la que predomina, representando más de la mitad de las intervenciones $(52 \%)$. Cuando discuten chicos y chicas por separado, destacan sobre todo las conversaciones de tipo acumulativo y exploratorio ( $38 \%$ y $36 \%$ ), y se reducen a la mitad las de tipo disputativo (24\%).

$\mathrm{Al}$ afrontar el dilema referido al conflicto de pandilla el mayor número de enunciados corresponde a los acumulativos en los grupos en los que chicos y chicas debaten por separado, representando dos terceras partes del total $(66 \%)$. Aunque también es el tipo de conversación más utilizado en los grupos de debate conjunto ante el mismo dilema, la frecuencia disminuye en esta situación (44\%), siendo relativamente similar a la modalidad disputativa (38\%). 
TABLA I

Recuento/Porcentaje tipo dilema, tipo grupo y modalidad conversacional

\begin{tabular}{|c|c|c|c|c|}
\hline & \multicolumn{3}{|c|}{ Modalidad Conversacional } & \multirow{2}{*}{ Total } \\
\hline & Acumulativa & Exploratoria & Disputativa & \\
\hline \multicolumn{5}{|l|}{ Conflicto Pandilla } \\
\hline Recuento & 237 & 76 & 42 & 355 \\
\hline Porcentaje & $66,80 \%$ & $21,40 \%$ & $11,80 \%$ & $100,00 \%$ \\
\hline \multicolumn{5}{|l|}{ 중 Juntos } \\
\hline Recuento & 194 & 79 & 168 & 441 \\
\hline Porcentaje & $44,00 \%$ & $17,90 \%$ & $38,10 \%$ & $100,00 \%$ \\
\hline \multicolumn{5}{|l|}{ Total } \\
\hline Recuento & 431 & 155 & 210 & 796 \\
\hline Porcentaje & $54,10 \%$ & $19,50 \%$ & $26,40 \%$ & $100,00 \%$ \\
\hline \multicolumn{5}{|l|}{ Violencia Pareja } \\
\hline $\begin{array}{ll}\text { ¿’o Separados } \\
\text { Recuento }\end{array}$ & 272 & 257 & 174 & 703 \\
\hline Porcentaje & $38,70 \%$ & $36,55 \%$ & $24,75 \%$ & $100,00 \%$ \\
\hline \multicolumn{5}{|l|}{ 수 Juntos } \\
\hline Recuento & 303 & 103 & 441 & 847 \\
\hline Porcentaje & $35,77 \%$ & $12,16 \%$ & $52,07 \%$ & $100,00 \%$ \\
\hline \multicolumn{5}{|l|}{ Total } \\
\hline Recuento & 575 & 360 & 615 & 1550 \\
\hline Porcentaje & $37,10 \%$ & $23,22 \%$ & $39,68 \%$ & $100,00 \%$ \\
\hline
\end{tabular}

A continuación, en la tabla II se presentan los valores estadísticos y de probabilidad de los tres efectos entre las variables. Podemos verificar que todos ellos tienen un valor de $p<$ .050 , lo cual indica que tanto los efectos principales del tipo de dilema $(p=.000)$ y el tipo de grupo $(p=.000)$ así como su interacción $(p=.009)$, tienen un impacto significativo sobre la modalidad conversacional. El valor de $R^{2}$ para este modelo se situó en .072, lo cual indica, según Cohen (1988), un tamaño de efecto aceptable (moderado) en la explicación de los datos.

TABLA II

Análisis de la Varianza. Modalidad conversacional (UNIANOVA)

\begin{tabular}{lccccc}
\hline \multicolumn{1}{c}{ Fuente } & $\begin{array}{c}\text { Suma de } \\
\text { cuadrados }\end{array}$ & $g l$ & $\begin{array}{c}\text { Media } \\
\text { cuadrática }\end{array}$ & $F$ & $\begin{array}{c}\text { Significación } \\
(p)\end{array}$ \\
\hline Modelo corregido & 129,864 & 3 & 43,288 & 60,619 & .000 \\
Intersección & 7168,11 & 1 & 7168,11 & 10037,952 & .000 \\
Tipo Dilema & 51,506 & 1 & 51,506 & 72,127 & .000 \\
Tipo Grupo & 80,76 & 1 & 80,76 & 113,093 & .000 \\
Tipo Dilema*Tipo Grupo & 4,909 & 1 & 4,909 & 6,875 & .009 \\
\hline
\end{tabular}

Análisis cualitativo de los contenidos conversacionales

En la tabla III se visualizan los contenidos temáticos más relevantes generados durante la discusión y el debate sobre los dos dilemas. Además, se presenta el número de enunciados de cada contenido en función de los modos conversacionales.

El tema más comentado con diferencia en los grupos de discusión ha sido la exención de culpa que los chicos manifiestan en relación a su papel como desencadenante del suicidio. 
TABLA III

Contenidos según modalidad conversacional

\begin{tabular}{lcccc}
\hline & \multicolumn{3}{c}{ Modalidad Conversacional } & \multirow{2}{*}{ Total } \\
\cline { 2 - 4 } Contenidos & Acumulativa & Exploratoria & Disputativa & \\
\hline Exención de culpabilidad en los chicos & 1 & 15 & 43 & 59 \\
Temor perdida pareja y control & 27 & 2 & 8 & 37 \\
La votación para resolver & 24 & 7 & 0 & 31 \\
Separación privado-público en la pareja & 5 & 1 & 17 & 23 \\
Continuidad de pareja con maltrato por amor & 4 & 1 & 17 & 22 \\
Dificultad de comunicación en la pareja & 9 & 4 & 6 & 19 \\
Responsabilidad de las chicas en la agresión & 0 & 11 & 0 & 11 \\
\hline
\end{tabular}

Se exponen multitud de justificaciones, sobre todo de carácter disputativo, para evadir dicha responsabilidad, atribuyendo lo ocurrido a la casualidad, la familia, los amigos o a la chica. Ejemplo:

1375: ac: ipero que eso no es matar!

1376: am: es inducir a matar

1377: ac: tú puedes decir mil cosas que te hagan no ser culpable, hay muchas formas de decirlo

Dos temas tratados conversacionalmente de manera preferentemente acumulativa han sido el temor a la pérdida de la pareja en las chicas y la votación para resolver la controversia en los chicos. Ilustramos el intento por preservar al chico en los enunciados 11191121, y el recurso de la mayoría entre el 228-231:

1119. be: ahora esto ahora lo otro, te preocupa más que se enfade

1120. er: temes perder más, perder antes, perder tu novio

1121. be: antes que a una amiga, es verdad, es que tu novio te puede dejar

228. an: vamos a darle un votillo de confianza

229. ju: y el que diga que no pues lo convencemos

230. ru: ahí va

231. am: pero es que votan todos ¿no? o ¿porque uno diga que no ya no va a venir?

Hay dos temas que se presentan de forma parecida en cuanto a número de enunciados y modo de conversación. Así, la separación de lo público y privado en la pareja (enunciados ejemplo 877-878) y la continuidad de una pareja con maltrato por amor (enunciados ejemplo 2494-2496), ambas manifestadas especialmente por las chicas, se abordan especialmente de un modo disputativo en términos conversacionales. Ejemplos:

877. ma: una pareja es cosa de dos

878. pa: claro, tú en una relación no te puedes meter

2494. ma: pero si estaba todo el día así pués se sentía mal la chavala

2495. fr: pués haberlo dejado y no se hubiese comido más la olla

2496. ma: bueno si lo quería pues no quería dejarlo

Cuando tratan el tema de la dificultad de comunicación dentro de una pareja no lo hacen de un modo conversacional específico. No obstante, destaca el tipo acumulativo (enunciados ejemplo 1099-1103):

1099. P: ¿con quién es más difícil hablar con una amiga con la que tienes un problema o con tu novio cuando tienes un problema?

1100. be: con tu novio

1101. es: con tu novio 
1102. ma: con el novio

1103. er: con tu novio

Finalmente, cuando debaten sobre la responsabilidad de la chica en la agresión lo hacen de manera exclusivamente exploratoria. Podemos observar entre los enunciados ejemplo 860-865 cómo tras exponer diferentes argumentos y razonamientos, las chicas llegan a un consenso o acuerdo y establecen un posicionamiento compartido:

860. ra: los dos tienen culpa porque la chica puede parar, puede dejarlo, si no quiere es porque no quiere 861. el: más el niño pero la niña también

862. pa: pero los amigos también querían que lo dejara

863. es: claro y ella era la que decía que no

864. mb: luego que la culpa es de los dos el por insultarla

865. pa: y ella por dejarse

\section{Discusión y conclusiones}

Recordemos que el objetivo general de este trabajo era el estudio de la gestión discursiva de episodios conflictivos de pandilla y violencia de pareja en adolescentes mediante el análisis de sus discursos durante el afrontamiento de dilemas. Este propósito lo alcanzamos mediante dos vías de acceso, es decir, estudiamos por un lado estadísticamente los efectos del tipo de grupo y del tipo de dilema sobre los modos conversacionales, y por otro, cualitativamente, las temáticas de contenido más relevantes surgidas debatiendo.

Referente al primer objetivo nuestros resultados muestran que los modos conversacionales empleados durante la gestión discursiva de los conflictos se han relacionado con el tipo de grupo y el tipo de dilema. Respecto a lo primero, existen diferencias conversacionales según discutan con compañeros del mismo género o de diferente, tal como planteaban Leman y Duveen (2003). Así, cuando chicos y chicas debaten juntos practican una conversación especialmente de carácter disputativo y conflictivo, con frecuentes imposiciones y mandatos, mientras que al hacerlo por separado predominan los discursos de tipo acumulativo y exploratorio, con colaboraciones, aceptación, razonamientos y resolución conjunta. Con respecto al tipo de dilema, hallamos variabilidad en función de si afrontan un episodio de conflicto (pandilla) o de violencia (pareja), haciéndose uso de un estilo conversacional acumulativo (de aceptación) y disputativo (de confrontación), respectivamente.

Recordemos la conveniencia de diferenciar el conflicto de la violencia para evitar el asociacionismo conceptual y la confusión terminológica (Pareja, 2007). Soriano (2009) destaca que el desenlace del conflicto depende de su gestión: si las estrategias son democráticas el conflicto se puede cerrar pacíficamente, pero si son demasiado agresivas suele transformarse en violencia. Así, el tránsito del conflicto a la violencia se evidencia cuando las diferentes formas de "percibir el mundo" dan lugar al uso de la fuerza y el poder como instrumentos de negación y rechazo de lo diferente. Esta transición puede estar mediatizada por las condiciones del contexto (Crandall et al., 1999; Juujärvi, 2006; Vikan, Camino y Biaggio, 2005). En nuestro trabajo ciertos elementos de la configuración grupal y el tipo de dilema han mostrado su relación con las respuestas disputativas e impositivas.

Además de los dos efectos principales se evidencia uno compuesto. Así, el debate se hace más extenso, disputativo y trabado cuando chicos y chicas afrontan juntos el problema de violencia de género, tal como hipotetizábamos para el primer objetivo, mientras que cuando discuten por separado sobre este mismo asunto hacen uso de una actitud discursiva que combina la tolerancia y aceptación con la negociación.

En relación a cómo tratan el tema de la violencia de género, el análisis de las temáticas de contenido realizado para nuestro segundo objetivo reveló que los chicos no reconocían lo sucedido como maltrato, eximiéndose de responsabilidad, situación que fue tratada de un modo conversacional disputativo, impositivo y conflictivo. Ferrer, Bosch, Ramis, Torres y Navarro (2006) señalan que las creencias y actitudes más tolerantes con la violen- 
cia hacia las mujeres constituyen uno de los factores de riesgo para su ocurrencia, y ello se explica por el manejo de mitos y neomitos sobre la violencia de género (Bosch y Ferrer, 2012; Lorente, 2009). Echeburúa, Amor y Fernández-Montalvo (2002) mencionan la existencia de estrategias para no sentirse responsables de lo ocurrido o evadir responsabilidades: negación del problema, minimización y atribución externa.

El fenómeno opuesto se ha evidenciado en las chicas, quienes posicionadas en el papel de víctimas y haciendo uso de un modo conversacional exploratorio, negociador y razonado acaban atribuyéndose parte de la responsabilidad, constatándose así diferencias de género al afrontar situaciones controvertidas (Sastre y Moreno, 2002; 2004). Recordemos que Gilligan (1993) defendía la existencia de una orientación hacia la responsabilidad o ética de cuidado especialmente, y no exclusivamente, en las chicas.

En nuestro estudio encontramos que los chicos adolescentes, mediante conversación de tipo acumulativo y con posiciones compartidas apelan a la votación, mecanismo que representa una regulación basada en el ejercicio de la mayoría y que se orienta por la ética de justicia. En cambio, la asunción de culpa y responsabilidad en las chicas pudiera estar indicando cómo hacen uso de elementos derivados de la ética de cuidado (Gilligan, 1982). Por lo tanto, y relacionado con nuestro segundo objetivo, encontramos indicios de diferencias entre chicos y chicas tanto en la manera de interpretar situaciones violentas como en las éticas subyacentes.

Skoe, Cumberland, Eisenberg, Hansen y Perry (2002) encontraron que las mujeres afrontan los conflictos cotidianos sobre todo en base a comportamientos y argumentos relacionados con el cuidado y mantenimiento de las relaciones, ocasionándoles un mayor grado de ansiedad personal. En nuestros datos encontramos algunas expresiones relacionadas con estos tipos de comportamientos. Así, las chicas han manifestado de modo acumulativo y compartido un evidente temor a la perdida de la pareja y dificultades para la comunicación en la misma, mientras que de forma disputativa y controvertida tratan la separación del ámbito privado de la pareja respecto al espacio público, el amor romántico (Bosch y Ferrer, 2003) y la dependencia emocional (Castelló, 2005) como mecanismos de fijación y mantenimiento de una pareja con maltrato.

De nuevo observamos la variación en los modos conversacionales, en esta ocasión en función del contenido temático discutido. A partir de ello se deduce que no se ha dado una gestión discursiva constante de resolución durante las situaciones problemáticas, sino que las respuestas se han visto condicionadas por las circunstancias particulares del tema debatido. Así, se constata la utilización de diferentes estrategias de regulación entre chicos y chicas afrontando el episodio de maltrato, por lo que la situación de violencia de pareja parece representar un ámbito de relación en el que se producen más diferencias en las respuestas argumentativas que ante el conflicto grupal. En este sentido pensamos que nuestros datos apuntan a una consideración del enunciado y del discurso como acciones situadas en contexto (Bakhtin, 1986). Autoras como Crawford (1995, 2003), Jaffee y Hyde (2000), Hyde (2005) afirman que se han exagerado las diferencias en el lenguaje de hombres y mujeres, dándose un efecto situado en contexto.

En este trabajo el análisis estadístico se he efectuado en base a las variables tipo de grupo y tipo de dilema, pero no en función del género. Por otro lado el análisis cualitativo nos ha permitido conocer cómo se manifiestan los temas y modos de conversación según el género pero no la incidencia del tipo de grupo y dilema. Por ello, en futuras investigaciones se debería compensar esta desigualdad y hacer los análisis cruzando estos tres elementos, lo que daría evidencias de posible diversidad conversacional en chicos y chicas que desmontara la tendencia a naturalizar las diferencias de sexo/género. Creemos que los resultados de nuestro estudio podrían optimizarse si mejoramos su diseño. Concretamente la elaboración de dilemas más homólogos podrían neutralizar posibles sesgos, es decir, que por un lado tanto en el conflicto de pandilla como en la violencia de pareja existan alternativamente un chico y una chica como protagonistas, y que por otro la gravedad de los hechos sea comparable en ambos. Asimismo, sería recomendable el control de la variable 
edad, ya que según la Estadística de Menores del INE (serie 2007/2011) se observan diferencias en adolescentes entre 14 y 17 años en indicadores relacionados con conflictos y violencia (delitos y faltas).

Consideramos que esta línea de investigación ofrece nuevas posibilidades y rutas de estudio. Su prospectiva se podría derivar hacia la observación del cambio de posturas durante el debate mediante la persuasión y empleo de técnicas retóricas. Igualmente, podríamos estudiar las secuencias de los turnos de conversación, es decir, delimitar la dirección del acto discursivo contemplando a quién se dirigen (a chico o a chica) específicamente cuando discuten.

De nuestro estudio se pueden delimitar algunas consideraciones e implicaciones de cara a la práctica profesional en el terreno educativo y de la formación. En primer lugar, queremos destacar el valor del discurso y del debate sobre dilemas como recurso que propicia el aprendizaje de destrezas sociales y comunicativas, requiere la exposición de puntos de vistas, permite acceder a nuevas ideas y buscar acuerdos aportando un nuevo modo de comprender la realidad y de entendimiento del otro. En segundo lugar el afrontamiento discursivo de un dilema representa una sucesión de actos de posicionamiento en relación a un tema concreto, que permite la manifestación de narrativas y relatos sobre la propia experiencia vital durante los intercambios conversacionales. Este aspecto a su vez contribuye al autoconocimiento y a la creación de la propia identidad (Bamberg, 2004, 2007; Davies y Harré, 1990), elemento crucial durante la adolescencia.

Encontramos también útil este trabajo para el estudio de las concepciones y creencias adolescentes en relación a la violencia de género y su repercusión. En este sentido, son de suma importancia las actividades dirigidas a la redefinición de los significados asociados al maltrato, aspecto que puede ser abordado mediante los usos discursivos y lingüísticos como mediadores de la acción humana (Wertsch, 1991). Bascón (2011) plantea una acción formativa con dos versiones homólogas dirigidas al profesorado y alumnado, cuyo objetivo es ofrecer un recurso orientado a entender y esclarecer el papel de los procesos psicológicos, especialmente el lenguaje, en los conflictos y la violencia en adolescentes. Por otro lado, se ha elaborado un sistema de indicadores y criterios de buenas prácticas en coeducación e igualdad (Rebollo et al., 2012), que sustentado en el enfoque Doing Gender (West y Zimmerman, 1987) certifica cada práctica como eficaz, adecuada, sostenible, legítima y replicable, con la finalidad de mejorar las relaciones entre adolescentes mediante el discurso y la conversación.

\section{Referencias}

Bakhtin, M. (1986). Speech genres and other late essays. Austin: University of Texas Press.

BAmberg, M. (2004). Considering counter narratives. En M. Bamberg \& A. Molly (Eds.) Considering counter narratives: Narrating, resisting, making sense (pp. 351-371). Amsterdam: John Benjamins.

BAmberg, M. (2007). Narrative, state of the art. Amsterdam: John Benjamins.

BAscón, M. J. (2011). Adolescencia y conflictos de género. Un estudio a través del discurso. Saarbrücken: Lap Lambert Academic Publishing GmbH \& Co. KG.

BILLIG, M. (1987). Arguing and thinking, a rhetorical approach to social psychology. Cambridge, MA: Cambridge University Press.

Bosch, E. \& Ferrer, V. A. (2003). Mujeres maltratadas: análisis de características sociodemográficas, de la relación de pareja y del maltrato. Intervención Psicosocial, 12 (3), 325-344.

Bosch, E. \& Ferrer, V. A. (2012). Nuevo mapa de los mitos sobre la violencia de género en el siglo XXI. Psicothema, 24 (4), 548-554.

Castelló, J. (2005). Dependencia emocional. Características y tratamiento. Madrid: Alianza Editorial.

Crandall, C. S., Tsang, A., Goldman, S., \& Pennington, J. T. (1999). News worthy moral dilemmas: justice, caring, and gender. Sex roles, 40 (3-4), 187-209.

CRAWFORD, M. (1995). Talking difference, on gender and language. Londres: Sage.

CRAWFORD, M. (2003). Gender and humor in societal context. Journal of pragmatics, 35, 1413-1430.

Crawford, M. \& Chaffin, R. (1997). The Meanings of Difference. Cognition in Social and Cultural Context. En P. J. Caplan, M. Crawford, J. S. Hyde \& J. T. Richarson (Eds.), Gender Differences in Human Cognition (pp. 81-130). Oxford: Oxford University Press. 
500 Infancia y Aprendizaje, 2013, 36 (4), pp. 489-506

Davies, B. \& Harré, R. (1990). Positioning: The Discursive Production of Selves. Journal for the Theory of Social Behaviour, 20, 43-63.

Díaz-Aguado, M. J. (2003). Adolescencia, sexismo y violencia de género. Papeles del psicólogo, 84, 35-44.

Echeburúa, E., Amor, P. \& Fernández-Montalvo, J. (2002). Vivir sin violencia. Aprender un nuevo estilo de vida. Madrid: Pirámide.

EDwards, D. (1996). Hacia una psicología discursiva de la educación en el aula. En C. Coll \& D. Edwards (Comps.) Enseñanza, aprendizaje y discurso en el aula. Aproximaciones al estudio del discurso educativo (pp. 3552). Madrid: Fundación Infancia y Aprendizaje.

Eyre, S. L., DAvis, E. W. \& PEACOCK, B. (2001). Moral argumentation in adolescents commentaries about sex. Culture, bealth E sexuality, 3, 1-17.

Ferrer, V. A., Bosch, E., Ramis, M. C., Torres, G. \& Navarro, C. (2006). La violencia contra las mujeres en la pareja: creencias y actitudes en estudiantes universitarios/as. Psicothema, 18 (3), 359-366.

GARAIGORDOBIL, M. (2012). Cooperative conflict-solving during adolescence: relationships with cognitive behavioural and predictor variables. Infancia y Aprendizaje, 35 (2), 151-165.

GILLIGAN, C. (1982). In a different voice: psychological theory and women's development. Cambridge, MA: Harvard University Press.

Gilligan, C. (1993). Reply to critics. En M. J. Larrabee (Ed.), An Ethic of Care (pp. 209-230). Londres: Routledge.

HYDE, J. (2005). The gender similarities hypothesis. American psychologist, 60 (6), 581-592.

INE (2012). Estadística de Menores, Registro Central de Sentencias de Responsabilidad Penal, Ministerio de Justicia. Consultado el 26 de diciembre de 2012, de http://www.ine.es/inebmenu/mnu_justicia.htm

Inglés, C. J., Martínez-Monteagudo, M. C., Delgado, B., Torregrosa, M. S., Redondo, J., Benavides, G., García-Fernández, J. M., \& García-López, L. J. (2008). Prevalencia de la conducta agresiva, conducta prosocial y ansiedad social en una muestra de adolescentes españoles: un estudio comparativo. Infancia y Aprendizaje, 31 (4), 449-461.

JAFFEE, S. \& HYDE, J. (2000). Gender differences in moral orientation: A meta-analysis. Psychological bulletin, $126(5), 703-726$.

JARES, X. (2001). Educación y conflicto. Guía de educación para la convivencia. Madrid: Editorial Popular.

JuUJÄRVI, S. (2006). Care reasoning in real-life moral conflicts. Journal of moral education, 35 (2), 197-211.

KuHN, D., Wang, Y. \& Li, H. (2010). Why Argue? Developing Understanding of the Purposes and Values of Argumentive Discourse. Discourse processes 48 (1), 26-49.

LEMAN, P. J. \& DuveEN, G. (2003). Gender identity, social influence and children's arguments. Swiss journal of psychology, 62 (3), 149-158.

LORENTE, M. (2009). Los nuevos hombres nuevos. Barcelona: Destino.

Mercer, N. (2001). Palabras y mentes. Cómo usamos el lenguaje para pensar juntos. Barcelona: Paidós.

Ortega, R. \& Mora-Merchán, J. A. (2008). Las redes de iguales y el fenómeno del acoso escolar: explorando el esquema dominio-sumisión. Infancia y Aprendizaje, 31 (4), 515-528.

Pareja, J. A. (2007). Conflicto, comunicación y liderazgo escolar: los vértices de un triángulo equilátero. Profesorado. Revista de curriculum y formación del profesorado, 11 (3), 1-19.

Potter, J., Wetherell, M. (1995). Discourse analysis. En J. A. Smith, R. Harré \& L. V. Langenhove (Eds.), Retbinking methods in psychology (pp. 80-92). Londres: Sage.

Prieto, M. T., Carrillo, J. C. \& JimÉnez, J. (2005). La violencia escolar. Un estudio en el nivel medio superior. Revista Mexicana de Investigación Educativa, 10 (27), 1027-1045.

QUignARD, M. (2005). A collaborative model in dyadic problem-solving interactions. En F. H. Van Eemeren \& P. Houtlosser (Eds.), Argumentation in practice (pp. 69-86). Filadelfia: John Benjamins Publishing Company.

Rebollo, M. A., Piedra, J., Sala, A., Sabuco, A., SaAvedra, F. J. \& Bascón, M. J. (2012). La equidad de género en educación: Análisis y descripción de buenas prácticas en educación. Revista de Educación, 358, 129-152.

Sastre, G., Arantes, V. \& GonZÁlez, A. (2007). Violencia contra las mujeres: significados cognitivos y afectivos en las representaciones mentales de adolescentes. Infancia y Aprendizaje, 30 (2), 197-213.

SASTRE, G. \& MORENO, M. (2002). Resolución de conflictos y aprendizaje emocional. Una perspectiva de género. Barcelona: Gedisa

SASTRE, G. \& MOREnO, M. (2004). Una perspectiva de género sobre conflictos y violencia. En E. Barberá \& I. Martínez-Benlloch (Coords.), Psicología y Género (pp. 121-143). Madrid: Pearson.

Skoe, E. A., Cumberland, A., Eisenberg, N., Hansen, K. \& Perry, J. (2002). The influences of sex and gender-role identity on moral cognition and prosocial personality traits. Sex Roles, 46 (9/10), 295-309.

SORIANO, A. (2009). Violencia y conflicto. La escuela como espacio de paz. Profesorado. Revista de curriculum y formación del profesorado, 13 (1), 321-334.

Vikan, A., CAMINO, C. \& BiagGIO, A. (2005). Note on a cross-cultural test of Gilligan's ethic of care. Journal of moral education, 34 (1), 107-111.

WERTSCH, J. (1991). Voices of the mind. A sociocultural approach to mediated action. Harvard: Harvard University Press.

West, C. \& Zimmerman, D. (1987). Doing Gender. Gender and Society, 1 (2), 125-151. 


\title{
Apéndice
}

\author{
Contenidos y modos conversacionales en adolescentes. Debatiendo sobre conflictos \\ grupales y violencia de pareja
}

\author{
Relatorio de cambios
}

A continuación presentamos la explicación de los cambios realizados ante las sugerencias de los/as revisores/as Para ello incluimos la propuesta de cambio (en negrita) y seguidamente la explicación de la modificación realizada en el artículo.

Revisor A

1. Expresiones como "pensamiento colectivo" muy usadas en los modelos cualitativos, son sin embargo confusas. Colectivamente se discute, se construyen acuerdos, consensos, disensos, etc. dejemos el pensamiento para como proceso básico psicológico. Recomiendo rectificar esa expresión. Eliminación y sustitución de la expresión "pensamiento colectivo": "En su afán por el estudio de los mecanismos de construcción compartida de significados durante la discusión propone la existencia de tres modos de conversación...” (pág. 4).

2. Resolución del episodio. No queda claro cuando se considera que se ha "resuelto el episodio". No se dice cuando se da por concluida la argumentación, ni que se considera resolución del episodio. Redactar mejor esta frase (última antes del método). Se sustituye la frase y queda así: “...dificultará la resolución del episodio, es decir, la consecución negociada de una postura relativamente compartida.” (pág. 6).

3. Conclusiones: la deriva hacia el análisis del "género" en las conclusiones no se corresponde necesariamente con la posición teórica que sabiamente se ha empleado como marco en la introducción. Constructos como conflicto y violencia entre iguales y en pareja adolescente que se supone son el fundamento del artículo quedan escasamente discutidos frente a la presencia del asunto "género" (legítimo) que recibe un tratamiento más extenso y analítico. Simplemente hay que no descuidar lo que en la introducción se ha considerado el sustrato teórico básico y relacionar los resultados con ello. En general, en este apartado se han intentado equilibrar los contenidos tratados en la introducción y la discusión, añadiendo en la discusión el tema conflicto-violencia y en la introducción el tema género (ambos faltaban). Concretamente, se conectan en la discusión los temas básicos planteados en la introducción (conflicto y violencia) con los hallazgos encontrados, dando un mayor peso al tratamiento e interpretación de estos dos conceptos: "Con respecto al tipo de dilema, hallamos variabilidad en función de si afrontan un episodio de conflicto (pandilla) o de violencia (pareja), haciéndose uso de un estilo conversacional acumulativo (de aceptación) y disputativo (de confrontación), respectivamente.

Recordemos la conveniencia de diferenciar el conflicto de la violencia para evitar el asociacionismo conceptual y la confusión terminológica (Pareja, 2007). Soriano (2009) destaca que el desenlace del conflicto depende de su gestión: si las estrategias son democráticas el conflicto se puede cerrar pacíficamente, pero si son demasiado agresivas suele transformarse en violencia. Así, el tránsito del conflicto a la violencia se evidencia cuando las diferentes formas de "percibir el mundo" dan lugar al uso de la fuerza y el poder como instrumentos de negación y rechazo de lo diferente. Esta transición puede estar mediatizada por las condiciones del contexto (Crandall et al., 1999; Vikan, Camino y Biaggio, 2005; Juujärvi, 2006). En nuestro trabajo ciertos elementos de la configuración grupal y el tipo de dilema han mostrado su relación con las respuestas disputativas e impositivas." (pág. 16); "Así, se constata la utilización de diferentes estrategias de regulación entre chicos y chicas afrontando el episodio de maltrato, por lo que la situación de violencia de pareja parece representar un ámbito de relación en el que se producen más diferencias en las respuestas argumentativas que ante el conflicto grupal' (pág. 18). Además de reorientar la discusión hacia la diferenciación del conflicto y la violencia en contextos socioculturales se procede a la eliminación de contenidos referentes al tema del género: "Autoras como Sastre y Moreno (2002, 2004) describen estas dos concepciones. La ética de justicia atribuye a todos los individuos los mismos deberes y derechos sin tener en cuenta la individualidad, mientras que la ética de cuidado es sensible a la historia singular y al componente emocional durante las situaciones interpersonales." (pág. 17, tras la mención a Gilligan). Se reducen y condensan contenidos sobre género: "Así, las chicas han manifestado de modo acumulativo y compartido un evidente temor a la perdida de la pareja y dificultades para la comunicación en la misma, mientras que de forma disputativa y controvertida tratan la separación del ámbito privado de la pareja respecto al espacio público, el amor romántico (Bosch y Ferrer, 2003) y la dependencia emocional (Castelló, 2005) como mecanismos de fijación y mantenimiento de una pareja con maltrato." (pág. 18). Asimismo, las principales y más relevantes perspectivas relativas el género tratadas en la discusión son incluidas en la introducción: "Resulta loable estudiar cómo se utilizan estos tres tipos de conversación durante la discusión de situaciones controvertidas. Así, por un lado parece interesante la influencia de aspectos como el tipo de problema abordado y el sexo, y por otro, los contenidos temáticos tratados. Con respecto a lo primero, Crandall, Tsang, Goldman y Pennington (1999) plantean la relación entre contenido de un dilema y usos discursivos, y Leman y Duveen (2003) entre estilo conversacional y sexo de la persona con la que se discute. Referente a las temáticas, Sastre, A rantes y González (2007) muestran cómo en la adolescencia no se identifica ni se interpreta claramente la violencia de género, produciéndose una tendencia a disculpar y mini- 
502 Infancia y Aprendizaje, 2013, 36 (4), pp. 489-506

mizar los actos violentos. Sobre este fenómeno, Díaz-Aguado (2003) observó que eran muchas más las chicas adolescentes que rechazaban el uso de la violencia y muchos más los chicos que lo justificaban culpando frecuentemente a la víctima.

Segun Gilligan (1982) estas diferencias en las formas de interpretar la violencia pudieran estar relacionadas con diferentes éticas, al observar la existencia de una orientación hacia la responsabilidad o ética de cuidado, preferentemente en las mujeres, que surge de la conciencia de formar parte de una red de relaciones y que adopta el punto de vista del "otro concreto", sus necesidades particulares, sentimientos y motivaciones. Esta manera de entender el mundo difiere de la ética de justicia que canaliza, mediante derechos y normas imparciales, sobre todo las acciones de los hombres. No obstante, la propia autora en una obra posterior (Gilligan, 1993) aclara que esta distinción no se sustenta en fundamentos esencialistas, sino que ambas éticas se complementan, relacionan e integran de cara al desarrollo.

Queremos resaltar que la utilización de la variable sexo podría inducir a una visión esencialista, homogénea y dicotómica. Por el contrario, adoptamos el enfoque Doing Gender (West y Zimmerman, 1987) que enfatiza la construcción social del género desde niveles socioculturales, relacionales e individuales, desencadenándose una "profecía autocumplida" (Crawfordy Chaffin, 1997) o comportamiento en consonancia con lo esperado." (pág. 5).

Revisor $B$

1. Resultaría más claro adelantar en el texto la exposición de los objetivos fundamentales y a partir de ahí fundamentar el problema. Se adelantan los objetivos al principio del manuscrito con idea de hacer una presentación inicial del propósito del trabajo: "El objetivo que guía este trabajo es analizar en adolescentes la gestión discursiva de situaciones interpersonales controvertidas, concretamente episodios de conflicto en grupos de iguales y de violencia de pareja, explorando los modos conversacionales y los contenidos que subyacen y orientan la conversación durante el afrontamiento de los mismos" (pág. 2).

2. Respecto al marco teórico, señalamos dos cuestiones. La primera se refiere a la presentación de la adolescencia como un periodo relacionado con los conflictos y la violencia. Nos parece que habría que reflexionar sobre la posibilidad de no adherirse a una representación de la adolescencia que, por un lado, abona la explicación de los comportamientos en clave individualista y, por otro, es parcial a la vista de los resultados empíricos y las reflexiones teóricas. Se matiza y aclara la relación entre adolescencia, conflicto y violencia considerando esta etapa como una fase evolutiva multidimensional, en la que pueden entrar en juego números factores de distinta naturaleza (individual, social, cultural, grupal, etc.) que originen episodios de conflicto y violencia, y que por tanto no se explica únicamente por determinantes individuales: "El tránsito por la adolescencia puede verse condicionado por factores de riesgo de distinta índole y procedencia: culturales, ambientales, relacionales, personales, etc., que a veces desembocan en conflictos y violencia" (pág. 2). Se concreta el foco del trabajo: "En este trabajo nos centramos principalmente en situaciones interpersonales como los conflictos de grupo y la violencia de pareja." (pág. 2).

La segunda cuestión se refiere a la utilización de la variable sexo. Al conformar dos grupos de acuerdo con el sexo-mujer/bombre- se organiza de nuevo la realidad a partir de una dicotomía y se induce a pesar en categorías homogéneas y visiones esencialistas. Es decir, si son mujeres (bombres), actuarán, conversarán de un modo particular, relacionado con su "naturaleza". Esto se ve reforzado al denominar al grupo "bomogéneo", es decir, se supone que entre las chicas o chicos no habrá diversidad. Esta visión resulta incongruente con la perspectiva de "Doing gender" recogida en las conclusiones. Se plantea el enfoque Doing Gender en la introducción como perspectiva que no sustentada en fundamentos esencialistas, sino que aboga por el proceso constructivo del género: "Queremos resaltar que la utilización de la variable sexo puede inducir a una visión esencialista, homogénea y dicotómica. Por contra adoptamos el enfoque Doing Gender (West y Zimmerman, 1987) que enfatiza la construcción social del género desde niveles socioculturales, relacionales e individuales, desencadenándose una "profecía autocumplida" (Crawford y Chaffin, 1997). En nuestro trabajo la separación de chicos y chicas en algunos grupos de discusión nos permitirá precisamente observar cómo se construyen y reproducen comportamientos en consonancia con lo esperado en uno y otro sexo." (pág. 6). Se modifica el nombre de las dos condiciones de la variable tipo de grupo (bomogéneo vs. mixto) por otras (chicos-chicas debatiendo por separado vs. chicos-chicas debatiendo juntos) para evitar una posición esencialista, especialmente, en la denominación del grupo bomogéneo que induce a no contemplar la diversidad dentro del propio sexo. Se procede al cambio de nomenclatura en todas las expresiones referentes a ello del manuscrito, incluida la Tabla I: "Modos conversacionales. Diseño y variables del análisis estadístico. Se consideraron tres variables; el tipo de grupo con dos condiciones, chicas-chicos debatiendo por separado y chicas-chicos debatiendo juntos, el tipo de dilema con otras dos condiciones, conflicto de pandilla y violencia de pareja, y la modalidad conversacional con tres categorías de análisis derivadas de la propuesta de Mercer (2001):" (pág. 10); "En cuatro grupos, chicas (dos) y chicos (dos) debatieron por separado, y en otros cuatro lo bicieron juntos." (pág. 9); "Los datos combinados entre las tres variables (tabla I) indican que la mayor producción discursiva tiene lugar cuando los y las adolescentes afrontan el dilema de violencia de género en los grupos en los que debaten juntos (frecuencia total=847). En estos escenarios, es principalmente la conversación disputativa la que predomina, representando más de la mitad de las intervenciones (52\%). Cuando discuten chicos y chicas por separado, destacan sobre todo las conversaciones de tipo acumulativo y exploratorio (38\% y 36\%), y se reducen a la mitad las de tipo disputativo (24\%)." (pág. 12); "Al afrontar el dilema referido al conflicto de pandilla el mayor número de enunciados corresponde a los acumulativos en los grupos en los que chicos y chicas debaten por separado, representado dos terceras partes del total (66\%). Aunque también es el tipo de conversación más utilizado en los grupos de debate conjunto ante el mismo dilema, la frecuencia disminuye en esta situación (44\%), siendo relativamente similar a la modalidad disputativa (38\%)." (pág. 12); Tabla I "సo+ Separados, ํํ Juntos" (pág. 26). 
3. La fundamentación del problema es correcta. Sin embargo, no vemos fundamentadas las hipótesis sobre el efecto de las variables tipo de grupo y tipo de dilema. Puede deberse a que su enunciación no es suficientemente rigurosa. Por ejemplo, la segunda bipótesis no nos parece tal a la luz de su enunciación"sospechamos"- y, fundamentalmente, de su contenido: "la mayoría de los contenidos y temáticas conversacionales van a girar sobre las relaciones de pareja y otros aspectos vinculados con las mismas". ¿De qué otro asunto se puede tratar dado el contenido de las situaciones? Modificación de la parte final del resumen adaptando su contenido al nuevo planteamiento del segundo objetivo: "Referente a los contenidos, se observan mayores diferencias temáticas entre chicos y chicas ante la violencia de pareja, evidenciándose el carácter situado del discurso; así, los chicos no visibilizan el maltrato mientras que las chicas asumen responsabilidad." (pág. 1). Se fundamentan y justifican teóricamente objetivos e hipótesis en la introducción: "Resulta loable estudiar cómo se utilizan estos tres tipos de conversación durante la discusión de situaciones controvertidas. Así, por un lado parece interesante la influencia de aspectos como el tipo de problema abordado y el sexo, y por otro, los contenidos temáticos tratados. Con respecto a lo primero, Crandall, Tsang, Goldman y Pennington (1999) plantean la relación entre contenido de un dilema y usos discursivos, y Leman y Duveen (2003) entre estilo conversacional y sexo de la persona con la que se discute. Referente a las temáticas, Sastre, Arantes y González (2007) muestran cómo en la adolescencia no se identifica ni se interpreta claramente la violencia de género, produciéndose una tendencia a disculpar y minimizar los actos violentos. Sobre este fenómeno, Díaz-Aguado (2003) observó que eran muchas más las chicas adolescentes que rechazaban el uso de la violencia y muchos más los chicos que lo justificaban culpando frecuentemente a la víctima.

Según Gilligan (1982) estas diferencias en las formas de interpretar la violencia pudieran estar relacionadas con diferentes éticas, al observar la existencia de una orientación hacia la responsabilidad o ética de cuidado, preferentemente en las mujeres, que surge de la conciencia de formar parte de una red de relaciones y que adopta el punto de vista del "otro concreto", sus necesidades particulares, sentimientos y motivaciones. Esta manera de entender el mundo difiere de la ética de justicia que canaliza, mediante derechos y normas imparciales, sobre todo las acciones de los hombres. No obstante, la propia autora en una obra posterior (Gilligan, 1993) aclara que esta distinción no se sustenta en fundamentos esencialistas, sino que ambas éticas se complementan, relacionan e integran de cara al desarrollo." (pág. 5). Se concretan y depuran los enunciados de los dos objetivos y de la hipótesis formulada para el primero de ellos. Se sustituye la hipótesis del segundo por un objetivo específico: "En definitiva, el propósito general de este trabajo se orienta hacia explorar la gestión discursiva de episodios de conflicto de pandilla y de violencia de pareja en adolescentes. Trataremos de aproximarnos mediante el estudio de los usos conversacionales de chicos y chicas adolescentes, durante la discusión y debate sobre dilemas. Se establecen dos objetivos: analizar el posible efecto del tipo de grupo y del tipo de dilema sobre los modos conversacionales (acumulativo, disputativo y exploratorio), e identificar los principales contenidos temáticos manifestados por chicos y chicas.

Para dar respuesta al primer objetivo planteamos la siguiente hipótesis: esperamos encontrar efectos significativos del tipo de grupo y del tipo de dilema sobre los modos conversacionales. Concretamente, un mayor número de intervenciones de tipo disputativo en los grupos de chicos y chicas debatiendo juntos sobre violencia de pareja, debido a diferencias en la interpretación y visibilización del maltrato de género; ello dificultará la resolución del episodio, es decir, la consecución negociada de una postura relativamente compartida. Respecto al segundo objetivo, queremos comprobar si las diferencias en las temáticas tratadas por chicos y chicas pueden asociarse con diferentes maneras de interpretar situaciones de violencia y/o con diferencias en las éticas subyacentes." (pág. 6).

4. En el apartado de metodología, la descripción de la muestra peca de superficial y nos surgen algunas preguntas. Se ha procedido a la reorganización de los apartados del método, quedando ahora en el siguiente orden: Participantes (pág. 7), Materiales (pág. 7), Procedimiento (pág. 9), Análisis (pág. 10).

¿Por qué el número de chicas y chicos es diferente, 23 chicas y 19 chicos? Se estudiaron dos aulas completas, correspondiendo estas cifras al resultante de los chicos y chicas de ambas; las diferencias se deben al mayor número de chicas en estas aulas. Trabajamos con la totalidad de alumnado de cada aula para evitar los posibles efectos negativos del descarte entre los participantes. El texto se modifica quedando así: "Participaron en el estudio 42 adolescentes de nacionalidad española correspondientes a dos aulas completas, 23 chicas y 19 chicos. La diferencia en el número se debió a la mayor presencia de chicas en las aulas y a las ausencias el día de la recogida de datos; además se evitó igualar los números mediante descartes por sus posibles efectos negativos." (pág. 7).

¿Por qué, dado el tamaño de la muestra, no se ha elegido un intervalo de edad menor o una sola edad? Puede haber diferencias importantes en adolescentes de 14 y de 17 años. Como se ha dicho anteriormente, seleccionamos dos aulas completas correspondientes a dos cursos (tercero y cuarto de ESO) para tener una representación mayor: "Seleccionamos edades entre 14 y 17 años para tener una representación más amplia del rango de edad en el que parece incrementarse el número de situaciones conflictivas y violentas." (pág. 7). No obstante, se apunta en la discusión la posibilidad de mejorar el diseño mediante la neutralización de los efectos extraños de la variable edad: "Asimismo, sería recomendable el control de la variable edad, ya que según la Estadística de Menores del INE (serie 2007/2011) se observan diferencias en adolescentes entre 14 y 17 años en indicadores relacionados con conflictos y violencia (delitos y faltas)." (pág. 19).

¿Cuáles son las posiciones mostradas ante los temas señalados que permiten seleccionar? ¿Cómo se han establecido esas posiciones y cómo se han evaluado en la muestra? ¿Cuántas personas de la muestra se adscriben a esas posiciones? Corresponde a la Fase 1 descrita en el procedimiento, en la que se lleva a cabo una tarea para la exploración de ideas previas en relación a las cuestiones sobre conflicto de pandilla y violencia de pareja. Concretamente, se realizaron debates con la totalidad del grupo-clase en los que se plantearon noticias y situaciones relacionadas con estos dos temas, pidiéndose a chicos y chicas que opinaran y se posicionaran en relación a ellos. Para ubicar a los y las participantes, se midió el grado de representación del 
504 Infancia y Aprendizaje, 2013, 36 (4), pp. 489-506

conflicto y la violencia que osciló entre la identificación y la no visibilización, pasando por estados intermedios de percepción parcial de estas manifestaciones, lo que permitió que todo el alumnado quedase ubicado en uno u otro nivel: "Para la formación de los grupos de discusión los y las participantes fueron seleccionados sobre la base de un criterio de heterogeneidad en función de las posiciones mostradas ante una tarea de exploración de ideas previas sobre conflicto grupal y violencia de pareja (Fase 1 del procedimiento). Se realizaron debates con la totalidad del grupo-clase en los que se plantearon noticias y situaciones relacionadas con estos dos temas para que chicos y chicas opinaran sobre ellos. Las posiciones fueron medidas en función del grado de representación del conflicto y la violencia, que osciló entre la identificación y la no visibilización, pasando por estados intermedios de percepción parcial. Esta horquilla permitió que todo el alumnado quedase ubicado en uno u otro nivel, y sirvió de base para garantizar que los diferentes niveles de representación generaran mayor confrontación durante el debate." (pág. 7).

Respecto a las variables, el diseño no permite denominar al tipo de grupo y de dilema variables "explicativas". Se suprime de todo el manuscrito el término "variable explicativa" y "variable de respuesta": "Se consideraron tres variables; el tipo de grupo con dos condiciones: homogéneo (chicas o chicos) y mixto (chicas y chicos), el tipo de dilema, con otras dos condiciones: conflicto de pandilla y violencia de pareja, y la modalidad conversacional, con tres categorías de análisis derivadas de la propuesta de Mercer (2001):" (pág. 10); "Tras registrar qué tipos de conversación emplean los y las adolescentes categorizando cada enunciado como acumulativo, disputativo o exploratorio, establecemos la significación estadística de los efectos entre las variables, es decir, tipo de grupo y tipo de dilema sobre la modalidad conversacional." (pág. 11); "En primer lugar se exponen los datos relativos a la distribución agrupada de las tres variables, y en segundo lugar mostramos los valores estadísticos $(F)$ de los efectos así como su probabilidad ( $p$ )." (pág. 12); "A continuación, en la tabla II se presentan los valores estadísticos y de probabilidad de los tres efectos entre las variables." (pág. 13).

\section{Procedimiento}

Se alude a una formación de los grupos de la forma más heterogénea posible (para generar controversia). No tenemos datos específicos sobre esa formación y su heterogeneidad y esto puede influir en el modo conversacional elegido. Ya se ha explicado antes, en el apartado referente a cómo se seleccionaron los y las participantes por sus diferentes posiciones en cuanto al grado de representación del conflicto y la violencia. Corresponde a la Fase 1: "Fase 1. Exploración de ideas previas. Chicos y chicas realizaron una tarea de posicionamiento y opinión sobre acontecimientos y noticias relacionadas con los temas que se iban a tratar posteriormente en los debates grupales. Esta tarea permitió conocer los puntos de vista, ideas previas y diferente grado de representación-visibilización sobre los conflictos de pandilla y la violencia de género en adolescentes, y así llevar a cabo la formación de los grupos de discusión de la forma más heterogénea posible, de cara a generar controversia en el debate." (pág. 9).

No queda claro en el texto si el dilema de la pandilla tiene alternativamente como protagonista a una chica o/y a un chico y, si es así, cómo se presenta a los dos tipos de grupos. Existe una diferencia con el dilema sobre la cuestión de violencia de género en el que el maltratador es un varón. Podemos entender los motivos pero esta diferencia debe controlarse para entender posteriormente los resultados. Si unimos a esto que la gravedad de los hechos no es parangonable en ambos dilemas, nos encontramos con la nuevas variables extrañas que dificultarán conocer a qué se deben los resultados. El dilema de pandilla tuvo dos versiones, una en la que el protagonista es un chico y otra en la que es una chica. Cada versión se presentó a cuatro grupos, dos de debate por separado (uno de chicos y otro de chicas) y dos debatiendo juntos. El dilema de violencia de pareja tuvo solo a un chico como maltratador, y se administró a los ocho grupos, cuatro de debate por separado (dos de chicas y dos de chicos) y cuatro juntos: "Se manejaron dos versiones homólogas del dilema de pandilla, una con un chico como protagonista y otra con una chica. Cada versión se planteó a cuatro grupos diferentes, uno de chicos y otro de chicas debatiendo por separado y dos haciéndolo juntos. El dilema de violencia de pareja tenía únicamente a un chico como maltratador, y se administró a los ocho grupos, cuatro de un tipo (dos de chicas y dos de chicos por separado) y cuatro del otro (debatiendo juntos)." (pág. 8). Se argumenta el motivo por el cual se elaboran los dilemas con diferente grado de gravedad y protagonismo, y se ofrecen detalles de su elaboración: "Se elaboraron dos dilemas o situaciones hipotéticas pero verosímiles y cercanas a la cotidianidad adolescente. Uno hacía referencia a un conflicto de intereses en pandilla y el otro a violencia de género. Se procuró mayor gravedad en el desenlace del episodio de maltrato en pareja y que la persona agresora fuese solo un chico buscando aumentar su visibilización y el impacto emocional. La construcción de los dilemas se llevó a cabo teniendo en cuenta los contenidos más comentados en la Fase 1 del procedimiento y en base a la literatura científica." (pág. 7). Se apunta en la discusión la posibilidad de mejorar el diseño de los dilemas con la idea de neutralizar efectos debidos a posibles variables extrañas: "Creemos que los resultados de nuestro estudio podrían optimizarse si mejoramos su diseño. Concretamente la elaboración de dilemas más bomólogos podrían neutralizar posibles sesgos, es decir, que por un lado tanto en el conflicto de pandilla como en la violencia de pareja existan alternativamente un chico y una chica como protagonistas, y que por otro la gravedad de los hechos sea comparable en ambos." (pág. 19).

\section{Análisis de los resultados}

Respecto al análisis cuantitativo, produce escasos datos y poco novedosos. La limitación mayor proviene no obstante del diseño. Así la falta de control de las variables -señalada en el procedimientoinfluye en que no sea posible explicar a qué se deben los resultados. Así, en el dilema de la pandilla, el modo más utilizado es el acumulativo en ambos tipos de grupo. La diferencia con el otro dilema puede deberse al agente o a la diferencia de gravedad del asunto. Esta corrección puede explicarse por el mismo cambio comentado: "Creemos que los resultados de nuestro estudio podrían optimizarse si mejoramos su diseño. Concretamente la elaboración de dilemas más homólogos podrían neutralizar posibles sesgos, es decir, que por un lado tanto 
en el conflicto de pandilla como en la violencia de pareja existan alternativamente un chico y una chica como protagonistas, y que por otro la gravedad de los hechos sea comparable en ambos." (pág. 19).

Los resultados cualitativos tienen mayor interés aunque no sabemos tampoco con qué variables se relacionan. Se deja claro que las temáticas surgidas durante el análisis cualitativo de los contenidos conversacionales sólo son estratificadas en función de las tres modalidades conversacionales del análisis cuantitativo, y no con las variables o factores tipo de grupo y tipo de dilema: "Las temáticas son cruzadas con los tres modos conversacionales planteados en el análisis anterior." (pág. 11). A este respecto, en la discusión se propone su posible estudio futuro, que ayudaría a conocer modos de debatir según grupos y dilemas y con ello cuestionar la naturalización de las diferencias de género "En este trabajo el análisis estadístico se he efectuado en base a las variables tipo de grupo y tipo de dilema, pero no en función del género. Por otro lado el análisis cualitativo nos ha permitido conocer cómo se manifiestan los temas y modos de conversación según el género pero no la incidencia del tipo de grupo y dilema. Por ello, en futuras investigaciones se debería compensar esta desigualdad y hacer ambos análisis cruzando estos tres elementos, lo que daría evidencias de posible diversidad conversacional en chicos y chicas que desmontara la tendencia a naturalizar las diferencias de sexo/género." (pág. 19).

Por otro lado, la interpretación de que los varones manifiestan "exención de culpa" en la agresión resulta dudosa si se toma como dato el extracto de entrevista presentado pues lo que los varones parecen negar no es la agresión sino su papel como desencadenante del suicidio. Rectificación del comentario sobre la temática exención de culpabilidad en los chicos del análisis cualitativo, haciendo hincapié en la minimización del papel como desencadénate del suicidio y no en la agresión: "El tema más comentado con diferencia en los grupos de discusión ha sido la exención de culpa que los chicos manifiestan en relación a su papel como desencadenante del suicidio" (pág. 13).

Dentro de esta perspectiva cualitativa, se podría haber aprovechado para exponer ejemplos de diversidad de modos de debatir dentro de los grupos considerados homogéneos para ir en contra de esa naturalización de las diferencias sexuales/de género. "Por otro lado el análisis cualitativo nos ha permitido conocer cómo se manifiestan los temas y modos de conversación según el género pero no la incidencia del tipo de grupo y dilema. Por ello, en futuras investigaciones se debería compensar esta desigualdad y hacer los análisis cruzando estos tres elementos, lo que daría evidencias de posible diversidad conversacional en chicos y chicas que desmontara la tendencia a naturalizar las diferencias de sexo/género." (pág. 19).

7. Una parte de las conclusiones podría incluirse en el marco teórico. Como se ha referido anteriormente, se incluyen en la introducción contenidos sobre género para su posterior discusión: "Resulta loable estudiar cómo se utilizan estos tres tipos de conversación durante la discusión de situaciones controvertidas. Así, por un lado parece interesante la influencia de aspectos como el tipo de problema abordado y el sexo, y por otro, los contenidos temáticos tratados. Con respecto a lo primero, Crandall, Tsang, Goldman y Pennington (1999) plantean la relación entre contenido de un dilema y usos discursivos, y Leman y Duveen (2003) entre estilo conversacional y sexo de la persona con la que se discute. Referente a las temáticas, Sastre, Arantes y González (2007) muestran cómo en la adolescencia no se identifica ni se interpreta claramente la violencia de género, produciéndose una tendencia a disculpar y minimizar los actos violentos. Sobre este fenómeno, Díaz-A guado (2003) observó que eran muchas más las chicas adolescentes que rechazaban el uso de la violencia y muchos más los chicos que lo justificaban culpando frecuentemente a la víctima.

Según Gilligan (1982) estas diferencias en las formas de interpretar la violencia pudieran estar relacionadas con diferentes éticas, al observar la existencia de una orientación hacia la responsabilidad o ética de cuidado, preferentemente en las mujeres, que surge de la conciencia de formar parte de una red de relaciones y que adopta el punto de vista del "otro concreto", sus necesidades particulares, sentimientos y motivaciones. Esta manera de entender el mundo difiere de la ética de justicia que canaliza, mediante derechos y normas imparciales, sobre todo las acciones de los hombres. No obstante, la propia autora en una obra posterior (Gilligan, 1993) aclara que esta distinción no se sustenta en fundamentos esencialistas, sino que ambas éticas se complementan, relacionan e integran de cara al desarrollo.

Queremos resaltar que la utilización de la variable sexo podría inducir a una visión esencialista, homogénea y dicotómica. Por el contrario, adoptamos el enfoque Doing Gender (West y Zimmerman, 1987) que enfatiza la construcción social del género desde niveles socioculturales, relacionales e individuales, desencadenándose una "profecía autocumplida" (Crawfordy Chaffin, 1997) o comportamiento en consonancia con lo esperado." (pág. 5).

Respecto a la exposición de la teoría de C. Gilligan, nos parece que da lugar a equívocos sobre la intención de la autora e incita a malinterpretarla ya que, tal como se presenta, da lugar a pensar que parte de presupuestos esencialistas. Su obra posterior, no citada, aclara plenamente que ése no es su punto de vista. Se elimina de la discusión el párrafo y medio en el que se describe inadecuadamente la posición de la autora, y se sustituye por una explicación en la introducción y una mención en la discusión en las que se dice que no representa un planteamiento esencialista: "No obstante, la propia autora en una obra posterior (Gilligan, 1993) aclara que esta distinción no se sustenta en fundamentos esencialistas, sino que ambas éticas se complementan, relacionan e integran de cara al desarrollo." (pág. 5); "Recordemos que Gilligan (1993) defendía la existencia de una orientación hacia la responsabilidad oética de cuidado especialmente, y no exclusivamente, en las chicas."

Nueva bibliografía añadida (se mantienen todas las del primer manuscrito)

Bosch, E. \& Ferrer, V. A. (2012). Nuevo mapa de los mitos sobre la violencia de género en el siglo XXI. Psicothema, 24 (4), 548-554. 
506 Infancia y Aprendizaje, 2013, 36 (4), pp. 489-506

Crawford, M. \& Chaffin, R. (1997). The Meanings of Difference. Cognition in Social and Cultural Context. En P. J. Caplan, M. Crawford, J. S. Hyde \& J. T. Richarson (Eds.), Gender Differences in Human Cognition (pp. 81-130). Oxford: Oxford University Press.

GiLligan, C. (1993). Reply to critics. En M. J. Larrabee (Ed.), An Etbic of Care (pp. 209-230). Londres: Routledge.

INE (2012). Estadística de Menores, Registro Central de Sentencias de Responsabilidad Penal, Ministerio de Justicia. Consultado el 26 de diciembre de 2012, de http://www.ine.es/inebmenu/mnu_justicia.htm

JUUJÄRVI, S. (2006). Care reasoning in real-life moral conflicts. Journal of moral education, 35 (2), 197-211.

LORENTE, M. (2009). Los nuevos hombres nuevos. Barcelona: Destino.

ParejA, J. A. (2007). Conflicto, comunicación y liderazgo escolar: los vértices de un triángulo equilátero. Profesorado. Revista de curriculum y formación del profesorado, 11 (3), 1-19.

Soriano, A. (2009). Violencia y conflicto. La escuela como espacio de paz. Profesorado. Revista de curriculum y formación del profesorado, 13 (1), 321-334.

VIKAN, A., CAMINO, C. \& BIAGGIO, A. (2005). Note on a cross-cultural test of Gilligan's ethic of care. Journal of moral education, 34 (1), 107-111. 